\section{Virtual and Augmented Reality in Surgical Specialties. Past, Present and Future}

Sir,

In the current digital era, virtual reality (VR) and augmented reality $(A R)$ are two commonly used terms that are now finding their way into medicine. These are often confused and thought to be similar but, as a matter of fact, technologically they are very distinct entities. VR is the mimicry of real-life contents in an entirely virtual context; whereas, AR overlays additional information (usually in the form of 3D, contextually aware graphics) onto a real-world view. ${ }^{1}$ But simply, AR adds further layers of information to the real world; whereas VR blocks it out entirely. Within current digital medical training using simulation, VR is currently more prominently adapted in practice as compared to $A R$, although a future shift is proposed.

In the past two decades, surgery and its training have evolved. This can be attributed to the development of minimally invasive surgery and the change in learning curves associated with it. Rapid technological advances are contributing to this evolution.

In the past, surgery used to be an apprenticeship; trainees would watch the seniors operate and attempt to copy their techniques to develop their surgical skills. The ethos "see one, do one, teach one" was the foundation of the surgical trainee. Gradual introduction into the procedure under the command of the seniors was the mainstay of education with minimal practice occurring outside of the patients themselves. All the teaching, learning and consolidation of the experience was carried out on patients under supervision of the master trainer.

At present, with the introduction of minimally invasive surgery, trainers are unable to control and guide trainee's movements during the procedure and the mode of delivery of training and learning curve have shifted dramatically. At this point, surgical training started entering the era of VT to allow trainees to immerse themselves in real-life situations and gain proficiency before being allowed to operate on a patient. Simulation is now becoming part of the surgical curriculum in many international training systems. This practice has followed that of the aviation industry, which has set extremely high safety standards. VT remains a critical part of a pilot's training, ensuring the pilot is well prepared to manage any unforeseen circumstances. The competency of the pilots in these scenarios is rigorously tested, a concept that now needs to be applied to trainees to improve outcomes within a healthcare system. ${ }^{2}$

In the future, if a surgeon can visualise the obscured vital structures, this would significantly reduce intraoperative complication risks; hence, improving patient outcomes. For example, as technology develops, the ability to superimpose blood vessel and bile geography onto a real-world view of the liver would allow a safer and quicker procedure in a potentially complicated case. Such technology would be instrumental in safe surgical practice, not only training, as is the case with VR.

As technology develops rapidly, new ideas are constantly being trialed to improve patient outcomes. The initiation and safe introduction of AR technologies within surgery would prove a milestone in technological development. With the advancement of robotic surgery, the use of AR in its simplest form is being increasingly utilised. Senior surgeons are able to annotate on the screens and this is then visible to the trainee on the console, allowing them to be directed through the case.

The conservatism of the surgical world still remains the limiting factor in this transition into a new exciting world. In addition, growing costs of the developing technology put pressure on healthcare systems around the world. Due to financial pressures, the fund holders of the public and healthcare systems are reluctant to make the investment. In other healthcare systems, the end user has to bear the additional costs, hence making healthcare deliverymore expensive.

In summary, these initial developments and further implementation and progression of new ideas present a significant outlay, but long-term projections show them to be economically beneficial. The stumbling block remains between the healthcare providers and the investors. But as technology moves forward, becomes mature and more cost effective, there is hope that the medical profession will transition with it.

\section{CONFLICT OF INTEREST:}

Author declared no conflict of interest.

\section{AUTHOR'S CONTRIBUTION:}

Al: Involved in the design of the work, drafting and revising the manuscript and approving it for submission.

\section{REFERENCES}

1. Chavan SR. Augmented reality vs virtual reality differences and similarities. Int J Adv Res Com Eng Tech 2016; 5:1947-52.

2. Alaker M, Wynn GR, Arumpalam T. Virtucal reality training in laparoscopic surgery systematic review and meta-analysis virtucal reality training in laparoscopic surgery. Int J Surg 2016; 85-94.

\section{Ahmer Irfan}

Department of General Surgery, Johns Hopkins Hospital, Baltimore, MD, USA

Correspondence to: Dr. Ahmer Irfan, Department of General Surgery, Johns Hopkins Hospital, Baltimore, MD, USA

E-mail:ahmerirfan@googlemail.com

Received: May 25, 2019; Revised: July 10, 2019;

Accepted: July 10, 2019

DOI: https://doi.org/10.29271/jcpsp.2020.04.455 